\title{
New papulovesicular rash in the course of COVID-19 signaling viral reactivation
}

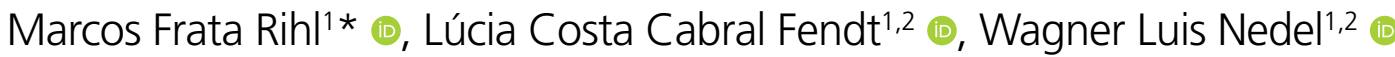

\section{SUMMARY}

Cutaneous manifestations are considered an infrequent presentation of coronavirus disease 2019 (COVID-19) and are mostly described in outpatient settings. Its onset during the course of the severe COVID-19 disease has been poorly described in severe cases. Studies focused on dermatological manifestations mostly described maculopapular or pernio-like lesions and less frequently vesicular or varicella-like eruption. We described the occurrence of a vesiculopapular eruption in three laboratoryconfirmed COVID-19 patients associated with severe lung injury in whom the skin findings preceded viral reactivation and recrudescence of hypoxemia. The potential mechanisms for COVID-19-related cutaneous manifestations include immune hypersensitivity, cytokine-release syndrome, deposition of microthrombi, and vasculitis.

KEYWORDS: Coronavirus infections. Severe acute respiratory syndrome. Dermatology. Exanthema. Recurrence.

The ongoing pandemic of coronavirus disease 2019 (COVID-19) is a global concern. The disease typically presents with symptoms resembling other viral infections, but in severe cases, it may develop acute respiratory distress syndrome with a high mortality rate ${ }^{1}$. Cutaneous manifestations are considered an infrequent presentation of COVID-19 infection but are probably under-recognized, although up to $44 \%$ of the patients had cutaneous findings at disease onset or during its course ${ }^{2}$. However, neither characteristics nor progression of the lesions were adequately documented ${ }^{2,3}$. Studies focused on dermatological manifestations have described different viral exanthems, of which vesicular eruptions appear in 9-11\% cases ${ }^{4,5}$. The majority of cases were recognized in outpatient settings, and although described in some severe cases, its onset over the course of the disease has not been established.

We describe the occurrence of a vesiculopapular eruption in three laboratory-confirmed COVID-19 patients (Figure 1) associated with severe lung injury in whom the skin findings preceded viral reactivation and recrudescence of hypoxemia, as a second hit of viral injury.

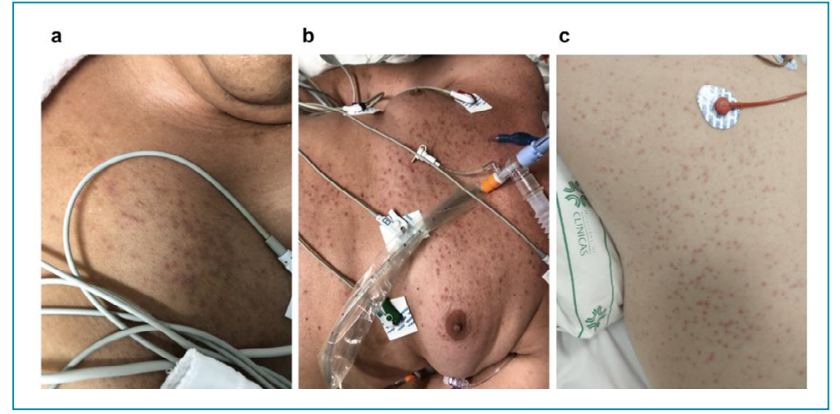

Figure 1. Papulovesicular eruptions before or concomitantly to second hit severe respiratory syndrome related to COVID-19 reactivation. (A) Case 1, (B) Case 2, and (C) Case 3.

${ }^{1}$ Hospital de Clínicas de Porto Alegre, Intensive Care Unit - Porto Alegre (RS), Brazil.

${ }^{2}$ Hospitalar Conceição, Intensive Care Unit - Porto Alegre (RS), Brazil.

*Corresponding author: marcosrihl@gmail.com

Conflicts of interest: the authors declare there are no conflicts of interest. Funding: none.

Received on October 22, 2020. Accepted on October 31, 2020. 


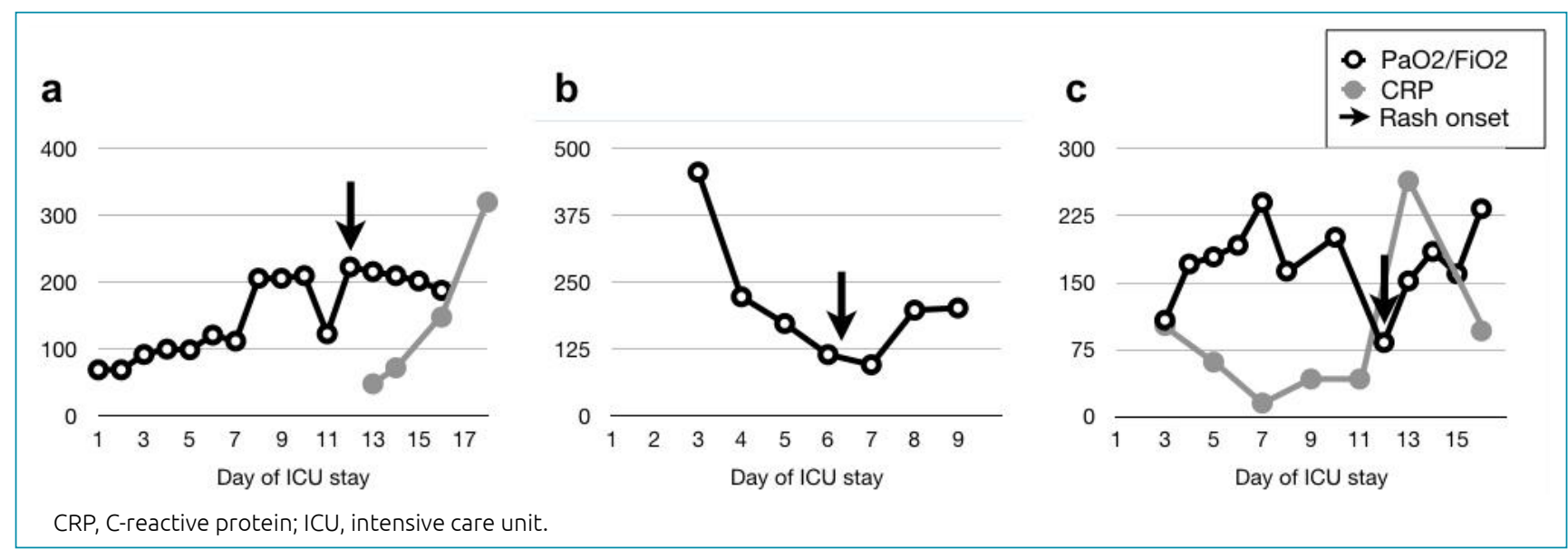

Figure 2. Graphs showing worsening in the $\mathrm{PaO}_{2} / \mathrm{FiO}_{2}$ ratio and raise of CRP after or concomitantly with the rash onset during the course of COVID-19 infection: (A) Case 1, (B) Case 2, and (C) Case 3.

\section{CASE 1}

A 58-year-old obese and diabetic female presented with 3-day COVID-19 symptoms and acute respiratory failure, requiring mechanical ventilation. Prone position was required for several days due to severe hypoxemia, with gradual improvement and progression to weaning. After 15 days, the patient developed a vesicular rash in the trunk, followed by new onset fever, severely worsening hypoxemia, and ascending C-reactive protein (CRP). Pulmonary embolism was ruled out, and no microbiological evidence of infection was found.

\section{CASE 2}

A 51-year-old male with diabetes history sought medical attention with symptoms of COVID-19 for 5 days, requiring invasive mechanical ventilation on the sixth day of hospital stay. One week later, the patient was in the process of weaning. He then developed a vesiculopapular rash followed by shock, fever, and loss of renal function, and over the following days, hypoxemia worsened. Blood and sputum cultures were negative. Computed tomography (CT) showed active viral lesions, with no evidence of pulmonary thromboembolism.

\section{CASE 3}

A 33-year-old obese male with ongoing viral symptoms for 1 week presented respiratory failure, requiring mechanical ventilation. After 11 days of intensive care, the patient showed signs of recovery and was weaned from the ventilator. The following day, he was reintubated facing worsening hypoxemia and simultaneously developed a vesicular rash affecting chest and back. CRP values increased significantly. Blood and sputum cultures were negative. The CT showed a small segmental pulmonary embolism and extensive ground-glass opacities compatible with viral activity.

In all three cases, the cutaneous eruption occurred either immediately before or concomitantly to second hit severe respiratory syndrome attributed to COVID-19 reactivation (Figure 2). Although a relation between vesicular skin eruption and disease recurrence has not yet been described, it is possible that inflammatory rebound and immune response phenomena could account for both features. The potential mechanisms for COVID-19-related cutaneous manifestations include immune hypersensitivity response to severe acute respiratory syndrome coronavirus 2 RNA, cytokine-release syndrome, deposition of microthrombi, and vasculitis ${ }^{6}$. Although further clinical data are warranted, new onset of papulovesicular rash might signal a more severe course of disease in patients with critical respiratory syndrome due to COVID-19.

\section{AUTHORS' CONTRIBUTIONS}

MFR: Conceptualization, Data Curation, Methodology, Writing - Original Draft, Writing - Review \& Editing. LCCF: Conceptualization, Data Curation, Methodology, Writing - Original Draft, Writing - Review \& Editing. WLN: Conceptualization, Data Curation, Methodology, Writing Original Draft, Writing - Review \& Editing. 


\section{REFERENCES}

1. Shang $Y$, Pan $C$, Yang $X$, Zhong $M$, Shang $X, W u Z$, et al. Management of critically ill patients with COVID-19 in ICU: statement from front-line intensive care experts in Wuhan, China. Ann Intensive Care. 2020;10(1):73. https:// doi.org/10.1186/s13613-020-00689-1

2. Gupta A, Madhavan MV, Sehgal K, Nair N, Mahajan S, Sehrawat TS, et al. Extrapulmonary manifestations of COVID-19. Nat Med. 2020;26(7):1017-32. https://doi.org/10.1038/s41591020-0968-3

3. Suchonwanit P, Leerunyakul K, Kositkuljorn C. Cutaneous manifestations in COVID-19: lessons learned from current evidence. J Am Acad Dermatol. 2020;83(1):e57-60. https:// doi.org/10.1016/j.jaad.2020.04.094
4. Freeman EE, McMahon DE, Lipoff JB, Rosenbach M, Kovarik C, Desai SR, et al. The spectrum of COVID-19-associated dermatologic manifestations: an international registry of 716 patients from 31 countries. J Am Acad Dermatol. 2020;83(4):111829. https://doi.org/10.1016/j.jaad.2020.06.1016

5. Casas CG, Català $A$, Hernández GC, Rodríguez-Jiménez $P$, Fernández-Nieto D, Rodríguez-Villa Lario A, et al. Classification of the cutaneous manifestations of COVID-19: a rapid prospective nationwide consensus study in Spain with 375 cases. Br J Dermatol. 2020;183(1):71-7. https://doi.org/10.1111/bjd.19163

6. Wei C, Friedman AJ. COVID-19 pandemic: are there unique cutaneous manifestations in patients infected with SARS-CoV-2? J Drugs Dermatol. 2020;19(5):554-5. PMID: 32484624 